\title{
New Fission Product Yield Measurements at Oregon State University
}

\author{
Aaron Tamashiro ${ }^{1, *}$, Jason Burke ${ }^{2}$, Stephen Padgett ${ }^{2}$, Sean Burcher ${ }^{2}$, Todd Palmer $^{1}$, and \\ Camille Palmer ${ }^{1}$ \\ ${ }^{1}$ Oregon State University \\ ${ }^{2}$ Lawrence Livermore National Laboratory
}

\begin{abstract}
Cumulative fast fission product yields for ${ }^{235} \mathrm{U}$ and ${ }^{238} \mathrm{U}$ were measured at Godiva-IV in burst mode. Data was collected starting 45 minutes after the prompt irradiation. Data analysis codes were developed to analyze gammarays and calculate fission product yields with their respective uncertainties. Due to uncertainties in the branching ratios, different $\gamma$-rays from the same isotope led to different fission yield. This led to an effort at Oregon State University (OSU) to utilize the Rabbit facility at their 1 MegaWatt Training, Research, Isotopes, General Atomics (TRIGA) nuclear research reactor. The new experimental setup will allow us to measure branching ratio data and short-lived fission product yields starting seconds after a prompt irradiation.
\end{abstract}

\section{Introduction}

Fission product yields are used in the prediction of the behavior and performance of engineered systems involving nuclear fission. Measurements of fission product yields have been traditionally performed through radiochemistry, mass spectrometry, and $\gamma$-ray spectroscopy, often with mono-energetic neutrons. Recently, the Godiva-IV critical assembly was employed to perform a burst irradiation of a sample of ${ }^{235} \mathrm{U}$ and ${ }^{238} \mathrm{U}$ with the goal of measuring fission yields within an hour of prompt irradiation of neutrons in the Watt fission spectrum. An experiment at OSU will measure fission yields seconds after a prompt irradiation. We have developed a $\gamma$ spectroscopy-based data analysis procedure that has been developed for the prediction of fission yields that is independent of the actinide sample for the Godiva-IV experiment and will be used for the OSU experiment.

\section{Godiva-IV Experiment}

Godiva-IV is a critical assembly composed of unreflected highly enriched uranium (HEU) metal fuel [1]. This device was intended to irradiate samples using prompt bursts of fast fission neutrons of around $24 \mu \mathrm{sec}$ in duration with a peak neutron flux of around $4 \times 10^{18}$ neutrons $/ \mathrm{cm}^{2} / \mathrm{sec}[1]$. Large neutron fluxes allow shorter irradiation times, minimizing neutron absorption in the subsequent fission products, and assuring larger fission product generation rates. Counting on the sample began 45 min after irradiation.

\footnotetext{
*e-mail: tamashia@oregonstate.edu
} 
Two Broad Energy Germanium (BEGe) detectors were used with a Lynx data acquisition (DAQ) system. The BEGe detectors were Canberra's model BE6530, which have a $60 \%$ relative efficiency. These were mounted on large cryostats filled with liquid nitrogen. The sample is placed $9 \mathrm{~cm}$ from each detector face. The $\gamma$-rays were detected by the BEGe spectrometers and were recorded in list mode format through the Lynx systems by Canberra's digital signal analyzer. The raw data is stored in separate files for each detector; the files contain a recorded time stamp and ADC channel for each $\gamma$-ray interaction. The detectors are synchronized, such that coincident events may be filtered out in the future, if needed. The resolution for the event clock timing was $100 \mathrm{~ns}$ and the resolution for the coincidence timing was $1 \mu \mathrm{s}$ FWHM.

\section{Data Analysis}

The fission product yield, $F P Y_{i}$, for isotope $i$ is calculated directly from the ratio of the number of decay events from isotope $i$ to the total number of fission events occured in an irradiation experiment. The total number of fission events $N_{f}$ is calculated using assuming a known reference fission product yield, $F P Y_{\text {ref }}$ :

$$
N_{f}=\left(\frac{A_{0}}{\epsilon_{\gamma} I}\right)\left[\frac{\tau_{1 / 2, r e f}}{\ln (2) F P Y_{r e f} R_{r e f}}\right] .
$$

The measured fission yield for isotope $i$ is written in terms of the reference isotope:

$$
F P Y_{i}=\frac{A_{0, i} \tau_{1 / 2, i}}{\ln (2) N_{f} \epsilon_{\gamma, i} I_{i} R_{i}}
$$

Here, $A_{0}$ is the initial activity of the induced radioactive fission product, $\epsilon_{\gamma}$ is the absolute detector efficiency, $I$ is the self attenuation of the $\gamma$-ray through the target, and $R$ is the branching ratio of the $\gamma$-ray observed. $\gamma$-ray detection efficiency was measured using standard calibration sources with known activities. The self attenuation of $\gamma$-rays within the sample is measured using known $\gamma$-rays produced by the decay of the actinide. The $\gamma$-ray activities from the fission products are measured in the experiment, while the other parameters are taken from ENDF-VIII.0 [2]. The reference fission product used to calculate the total number of fission events is ${ }^{99} \mathrm{Mo}$.

\subsection{Interfering $\gamma$-Rays}

To analyze the $\gamma$-ray peaks, references are needed to help identify which $\gamma$-ray peaks are from which decaying radioisotope. A reference library is compiled using data from ENDF-VIII.0 [2]. The compiled data assists the user in isolating the $\gamma$-ray of interest from other $\gamma$-rays nearby that would contribute into the activity of the $\gamma$-ray peak being analyzed.

\section{$3.2 \gamma$-Ray Peak Analysis}

The $\gamma$-ray peaks are analyzed with a code written in $\mathrm{C}++$ and utilizing the ROOT data analysis framework [3]. By defining unique energy intervals for each peak, the peaks are isolated in the spectrum so they can be analyzed independently. These intervals are determined visually using cascade plots [see Figure 1].

Figure 1 shows an example of a cascade plots for a $\gamma$-ray peak at $196 \mathrm{keV}$ associated with the fission product ${ }^{88} \mathrm{Kr}$. Each color represents a different time bin and as time progresses the 


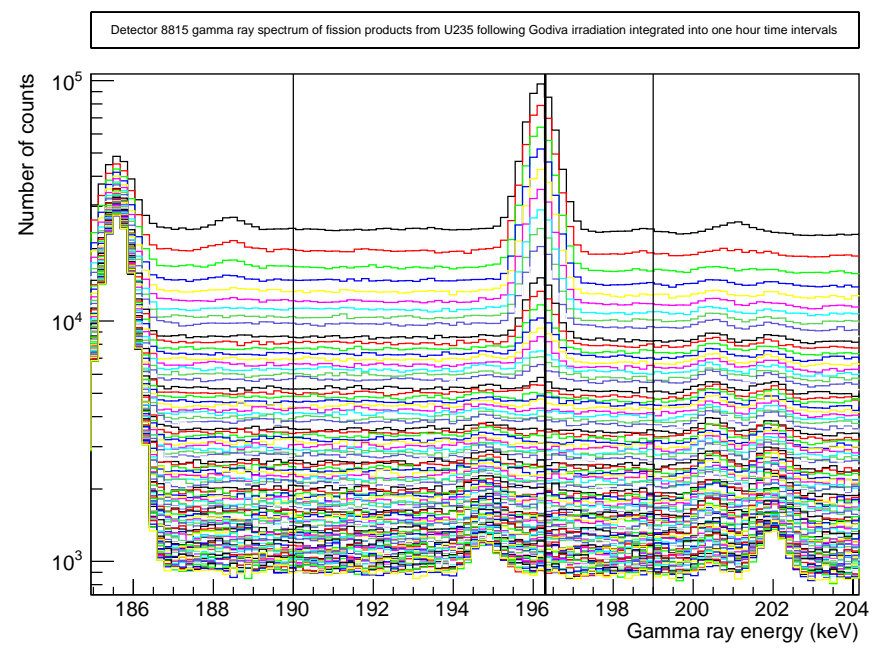

Figure $1 .{ }^{88} \mathrm{Kr} \gamma$-ray cascade plot for the $196 \mathrm{keV} \gamma$-ray

overall count rate decrease. In this example, the data collected in the first three time bins is used to set the energy interval to $[190.0 \mathrm{keV}, 199.0 \mathrm{keV}]$ to filter out the peaks near 188.5 $\mathrm{keV}$ and $201 \mathrm{keV}$. The interval chosen attempts to include as much background as possible; without including neighboring peaks.

As the time binned data is acquired, the live and raw time are used for the dead time correction by multiplying the counts for an energy bin with the ratio of raw time divided by live time. The ADC channel number is converted into $\gamma$-ray energy by using calibration sources. The time binned data are $2 \mathrm{D}$ histograms with $\mathrm{E}_{\gamma}$ on $\mathrm{x}$-axis and time on the $\mathrm{y}$ axis. Using this $2 \mathrm{D}$ histogram, a $\gamma$-ray spectrum for a specific time interval is produced by projecting the corresponding time bins on the x-axis. A Gaussian fit function with a linear background is used to fit peaks in the $\gamma$-ray spectrum:

$$
F(x)=C \times \exp \left[-\frac{(x-\mu)^{2}}{2 \sigma^{2}}\right]+m x+y
$$

where $C$ is the amplitude of the peak, $\mu$ is the energy of the peak, $\sigma$ is the resolution of the detector, $m$ is the slope of the background, $y$ is the y-intercept of the background, and $x$ is energy. This technique is used when there is a background continuum present [4]. Before the code fits every time slice, a master fit is produced to provide the initial fit parameters for the $\gamma$-ray peak [see Figure 2]. The histogram for the master fit is the produced by summing the data over all the time bins in the chosen time interval for each channel.

The linear background is subtracted out and the integral $S$ is the net counts in each respective time bin.

$$
\begin{aligned}
S & =\int\left(C \times \exp \left[-\frac{(x-\mu)^{2}}{2 \sigma^{2}}\right]+m x+y\right) d x-\int(m x+y) d x \\
& =\int\left(C \times \exp \left[-\frac{(x-\mu)^{2}}{2 \sigma^{2}}\right]\right) d x
\end{aligned}
$$




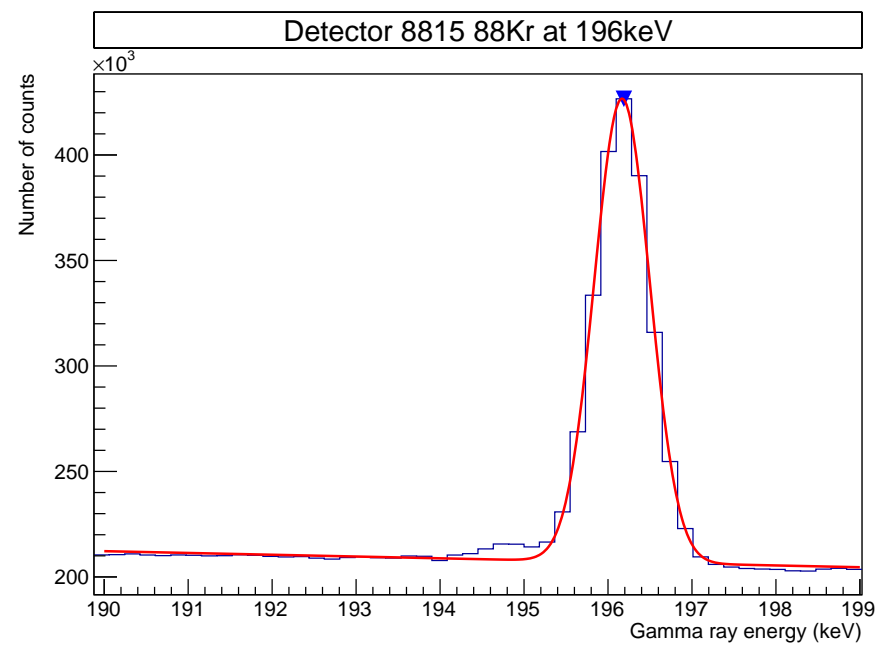

Figure 2. ${ }^{88} \mathrm{Kr}$ master sum plot fit for the $196 \mathrm{keV} \gamma$ ray

\subsection{Decay Curve Fit}

Net counts and half-life data are used to fit a decay curve that is extrapolated to time zero. The data for this curve consists of the counts integrated over each peak (using the energy interval established previously) in each time bin . To secure a proper decay curve, the half life and time increments were fixed. The only free parameter in the fit was the initial count rate $\left(A_{0}\right)$. Provided is the fit function:

$$
N(t)=\left(\frac{A_{0} \tau_{1 / 2}}{\ln (2)}\right)\left(\exp \left(-\frac{\ln (2)}{\tau_{1 / 2}} t\right)\right)\left(1-\exp \left(-\frac{\ln (2)}{\tau_{1 / 2}} \Delta t\right)\right)
$$

where $N(t)$ is the counts of $\gamma$-rays, $A_{0}$ is the initial count rate of $\gamma$-rays, $\tau_{1 / 2}$ is half life, $\Delta t$ is time step, and $t$ is time. This equation is derived from the integral of the count rate over a time bin.

Figure 3 is a plot of activity vs. time. The black points are the net counts with error for each time bin. The blue line is the fit function extrapolated over all time, the time interval used for the fit function is indicated by the highlighted thicker line on the plot.

In order to test the fit of the decay curve, a residual plot and $\chi_{v}^{2}$ plot are created. Residual plots are created to visualize the relative difference between the decay curve fit and the net count data in the time interval chosen. The residual plot is another criteria used to help determine an energy and time interval for the $\gamma$-ray analysis. The time interval is varied until residuals are less than a user-defined value of $20 \%$. The goal is to include as many points in the fit as possible, while minimizing the relative differences in the residual plot and keeping $\chi_{v}^{2}$ close to 1 .

\subsection{Fission Product Yield Calculation}

The initial count rate of $\gamma$-rays is passed on to a final code that uses Equation 2 to calculate fission product yield. The uncertainties were calculated using standard error propagation [5]. Different fission product yields from analyzing a $\gamma$-ray from a specific isotope compared to 


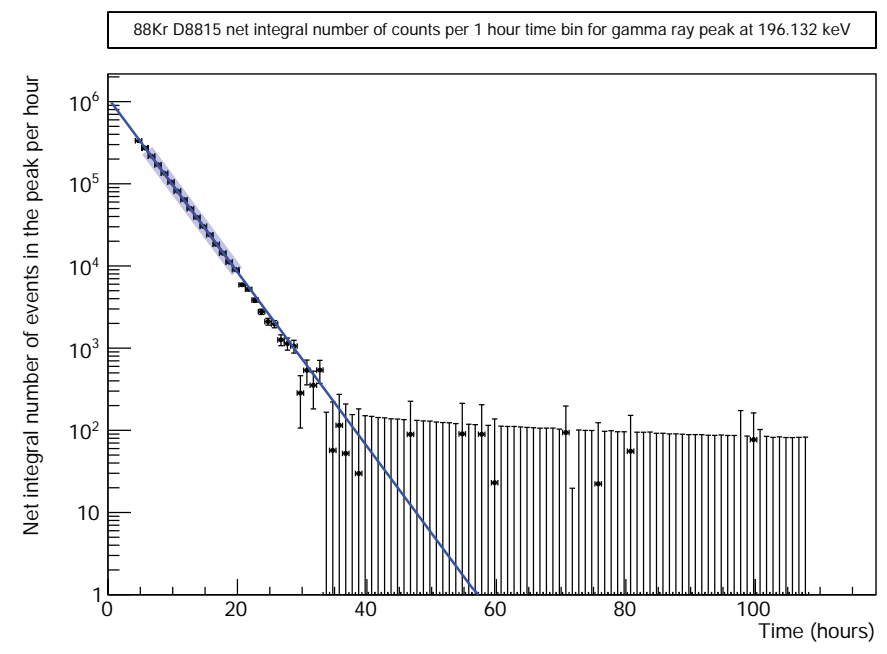

Figure 3. ${ }^{88} \mathrm{Kr} \gamma$ ray half-life plot for the $196 \mathrm{keV} \gamma$ ray

another $\gamma$-ray from the same isotope would imply there are large uncertainties in the $\gamma$-ray branching ratio data or there are unknown interferences. Further information may be found in reference [6].

\section{Oregon State University Test Run}

A proof of principle measurement was done at OSU using their TRIGA Reactor Rabbit system. A sample of ${ }^{238} \mathrm{U}$ was transported into the reactor core pneumatically and irradiated for 2 seconds. The sample is then counted in list mode and the data was parsed into 30 second time bins [see Figure 4]. This allows for the measurement of short-lived $\left(\tau_{1 / 2} \leq 1 \mathrm{hr}\right)$ fisison product yields. A future measurement is planned to evaluate independent fisison product yields and relative branching ratios.

\section{Conclusions}

The experimental setup was established in a room next door to the Godiva-IV critical assembly. The detection system and dimensions are all well established for future measurements. The data analysis routine is generalized and any modifications to the codes are easily applied. At least $100 \gamma$-rays from around 30 isotopes were analyzed for ${ }^{235} \mathrm{U}$ and ${ }^{238} \mathrm{U}$ Even though the codes are generalized for any $\gamma$-ray peak in the spectra, human input is still needed for each $\gamma$-ray in the choice of energy window and time interval for the data analysis.

Future work will involve improvements in the data analysis. The improvements will eliminate human intervention and fully automate the data analysis. Also, the experimental setup and data analysis will be applied to other actinides to analyze their fission product yields. Improvements in the branching ratio data will be worked out with $\gamma$ coincidence measurements at OSU. For Godiva-IV experiments, ${ }^{239} \mathrm{Pu}$ data is being analyzed and ${ }^{237} \mathrm{~Np}$ is planned.

\section{Acknowledgments}

This work was funded by the Office of Defense Nuclear Nonproliferation Research and Development within the U.S. Department of Energy's National Nuclear Security Administration 


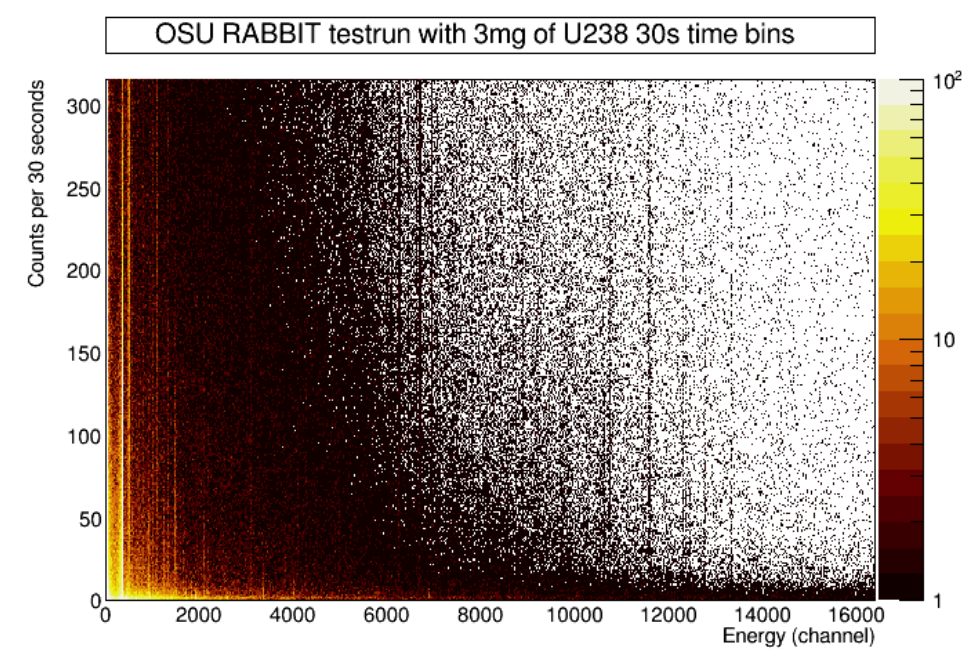

Figure 4. Test irradiation of U238 in the OSU TRIGA Rabbit

by Lawrence Livermore National Laboratory under Contract No DE-AC52-07NA27344. The U.S. Department of Energy's Nuclear Criticality Safety Programs National Criticality Experiments Research Center (NCERC), utilized in this work, is supported by the National Nuclear Security Administration's Office of the Chief of Defense Nuclear Safety, NA-511.

\section{References}

[1] R.E. Malenfant, Tech. rep. (1981)

[2] D.A. Brown et al., Nuclear Data Sheets 148, 2887 (2018)

[3] I. Antcheva et al., Computer Physics Communication 180, 2499 (2009)

[4] G.F. Knoll, Radiation Detection and Measurement (Wiley, 2010)

[5] P. Bevington, D.K. Robinson, Data Reduction and Error Analysis for the Physical Sciences (McGraw-Hill, 2010)

[6] A.S. Tamashiro, Master's thesis, Oregon State University (2019) 